SYMPOSIUM NO. 215

\title{
STELLAR ROTATION
}

Edited by: ANDRÉ MAEDER and PHILIPPE EENENS

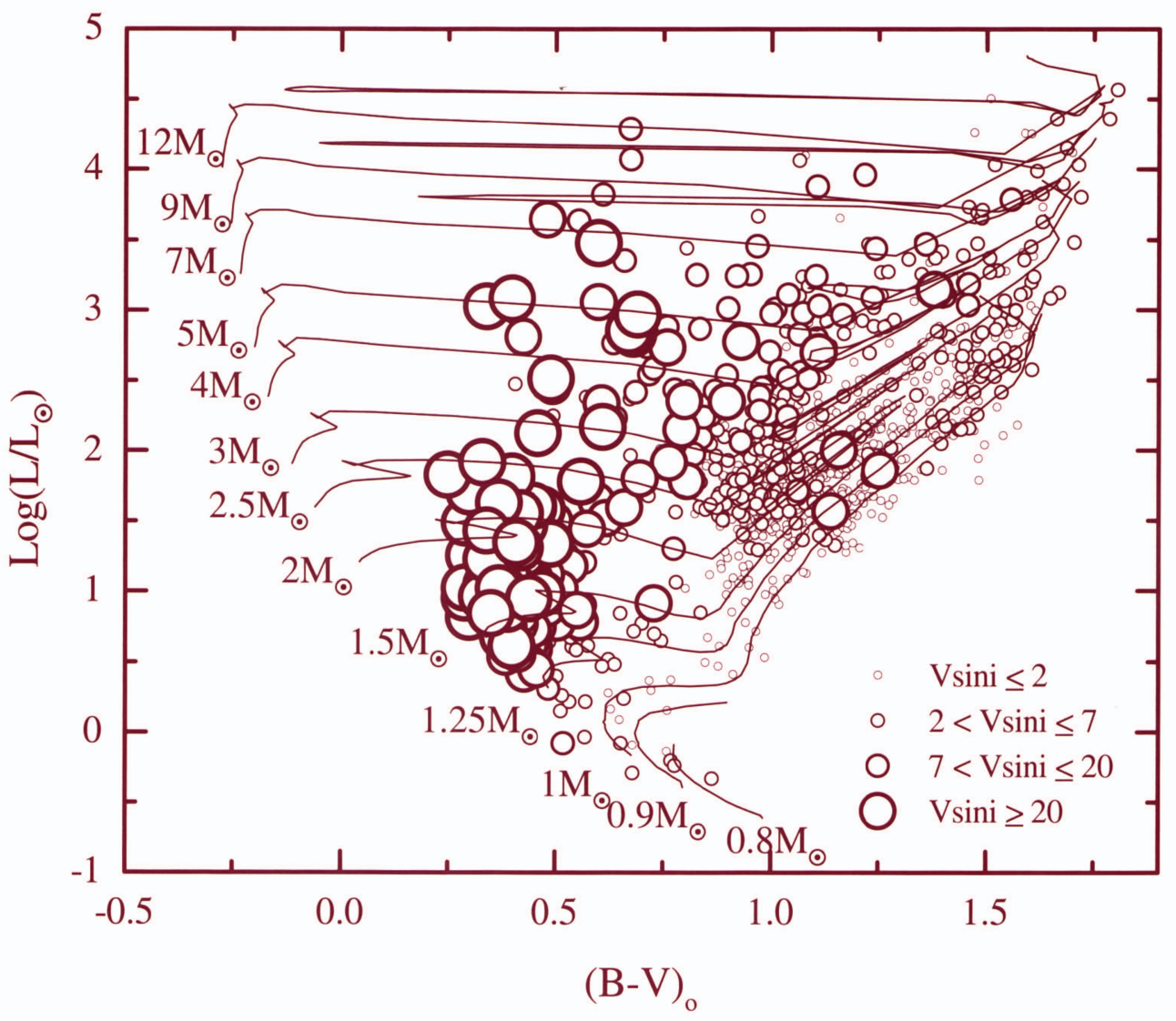

\section{$\Psi A \mathbb{A}$}

INTERNATIONAL ASTRONOMICAL UNION

PUBLISHER:

ASTRONOMICAL SOCIETY OF THE PACIFIC 
IAU SYMPOSIUM VOLUME 215

Cover Illustration:

Distribution of the rotational velocities of 2000 giant stars in the HR diagram (see Figure 1, page 147 -- paper by J. R. De Medeiros, page 144, this volume). 
ASTRONOMICAL SOCIETY OF THE PACIFIC 390 Ashton Avenue - San Francisco - California - USA 94112-1722 Phone: (415) 337-1100 Fax: (415) 337-5205 E-Mail: service@ astrosociety.org Web Site: www.astrosociety.org

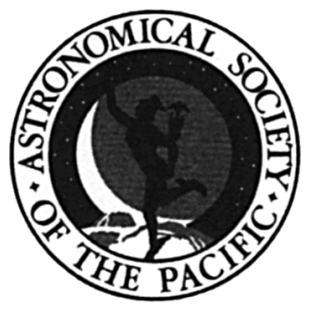

\section{ASP-CS VOLUMES \& IAU PUBLICATIONS - EDITORIAL STAFF}

Managing Editor: J. W. Moody

Publication Manager: Enid L. Livingston

PO Box 24463, Room 211 - KMB, Brigham Young University, Provo, Utah, 84602-4463 Phone: (801) 422-2111 Fax: (801) 422-0624 E-Mail: pasp@ byu.edu

LaTeX-Computer Consultant: T. J. Mahoney (Spain) - tjm@1l.iac.es

A listing of all other IAU Volumes published by

the Astronomical Society of the Pacific, is cited at the back of this volume 


\section{INTERNATIONAL ASTRONOMICAL UNION \\ 98bis, Bd Arago - F-75014 Paris - France \\ Tel: + +33143258358 E-mail: iau@iap.fr \\ Fax: +33 143252616 Web Site: www.iau.org}

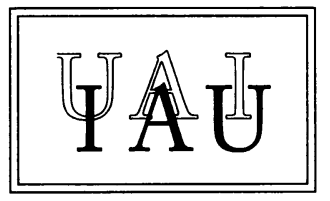

\section{STELLAR ROTATION}

Proceedings of the 215th Symposium

of the International Astronomical Union held at Hotel Westin, Cancun, Mexico

11-15 November 2002

Edited by

ANDRÉ MAEDER

Geneva Observatory, Geneva, Switzerland

and

\section{PHILIPPE EENENS}

Physics Department, University of Guanajuato, Guanajuato, Mexico 
(C) 2004 by International Astronomical Union All Rights Reserved

No part of the material protected by this copyright notice may be reproduced or utilized in any form or by any means - graphic, electronic, or mechanical including photocopying, taping, recording or by any information storage and retrieval system, without written permission from the IAU.

Library of Congress Cataloging in Publication Data

Main entry under title

LOC \#: $\quad 2004112685$

ISBN: $\quad 1-58381-180-\mathrm{X}$

IAU Publications - First Edition

Published on behalf of the IAU by: Astronomical Society of the Pacific

Printed in United States of America by Sheridan Books, Ann Arbor, Michigan 


\section{Contents}

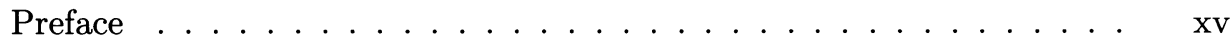

Conference Photograph $\ldots \ldots \ldots \ldots \ldots \ldots$ xvii

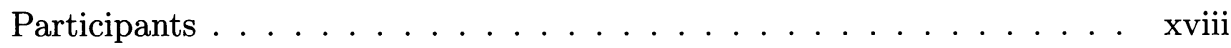

\section{SESSION 1. OBSERVATIONS OF ROTATING STARS}

\subsection{Determination of Rotation Velocities and Atmosphere Models}

The Determination and Interpretation of the Rotation Parameter $v \sin i$

(Invited Review) . . . . . . . . . . . . . . . 3

G.W. Collins, II

Advantages of the Broadening Function (BF) over the Cross-Correlation

Function $(\mathrm{CCF}) \ldots \ldots \ldots \ldots \ldots \ldots$

S.M. Rucinski

A Calibration of FWHM vs. $v$ for UV Lines . . . . . . . . .

I. Ramírez, G. Koenigsberger, R.L. Kurucz

Differential Rotation and the $v \sin i$ Parameter

J. Zorec, A. Domiciano de Souza, Y. Frémat

Fast Rotating Stars: Effect of the Aspect Angle on Line Profiles . . . .

Y. Frémat, J. Zorec, A.M. Hubert, M. Floquet, N. Leister,

$R$. Levenhagen, J. Chauville, D. Ballereau

NLTE Model Atmospheres with Rotation . . . . . . . . . . . .

D. Korčáková, J. Kubát

Absolute Wavelength Shifts - A New Diagnostic for Rapidly Rotating Stars

D. Dravins

\subsection{Rotation in O, B, A Stars and in Open Clusters}

Rotation and Line Broadening in OBA Stars (Invited Review) . . .

I.D. Howarth 
Projected Rotational Velocities in Galactic OB Stars of Different OB

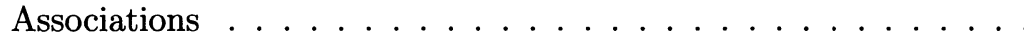

L.J. Corral, A. Herrero, M.R. Villamariz

A Search for Rotationally Modulated $\mathrm{H} \alpha$ Variability in OB Supergiants

T. Morel, S.V. Marchenko, A.K. Pati, K. Kuppuswamy, M.T. Carini,

E. Wood, R. Zimmerman

Observational Effects of Corotating Interaction Regions in OB stars . .

R. Blomme

The Possibility of a Disk Formation around Late B Stars . . . . . . .

J. Kubát, J. Krtička, I.B. Pustylnik, V. Votruba

New Projected Rotational Velocities of All Southern B-type Stars of the Bright Star Catalogue . . . . . . . . . . . . . .

H. Levato, M. Grosso

Rotational Velocities of B, A, and Early-F Stars . . . . . . . .

F.C. Fekel, P.B. Warner, A.B. Kaye

Rotational Velocity Distributions of A-Type Main-Sequence Stars . . .

F. Royer, J. Zorec, Y. Frémat

Stellar Rotation in Galactic Clusters (Invited Review) . . . . . . .

D.R. Gies, W. Huang

Stellar Rotation in the Young Cluster M17

W. Huang, D. Gies, P. Massey, P.S. Conti

New Homogeneous $v \sin i$ Determinations for B Stars in Galactic Open Clusters . . . . . . . . . . . . . . . .

P. North, F. Royer, C. Melo, J. R. de Medeiros, J. D. do Nascimento Jr., J.-C. Mermilliod, E. K. Grebel, A. Maeder, R. Mathieu

Metallicity and $v \sin i$ of B Stars in Galactic Open Clusters: is there any correlation ? . . . . . . . . . . . . . . F. Royer, C. Melo, J.-C. Mermilliod, P. North, J. D. do Nascimento Jr., J. R. de Medeiros, E. K. Grebel, A. Maeder

\subsection{Rotation in Be Stars, B[e] Stars and WR Stars}

Rotation and Properties of Be Stars (Invited Review) . . . . . . . 73

J. Zorec

The Initial Mass Function of Be Stars . . . . . . . . . . . . . .

$J$. Zorec, R. Levenhagen, J. Chauville, Y. Frémat, D. Ballereau,

A.M. Hubert, M. Floquet, N.V. Leister

Correlation between $v \sin i$ and the Peculiar Velocities of Be Stars

J. Zorec, J. Chauville, R. Levenhagen, N.V. Leister, D. Ballereau

Evolutionary State of "Field" Be Stars Deduced from BCD Parameters

J. Zorec, Y. Frémat, L. Cidale, A.M. Hubert, M. Floquet 
Rotational Velocities of "Field" Be Stars . . . . . . . . . . . . . .

J. Zorec, R. Levenhagen, J. Chauville, F. Royer, N.V. Leister, Y. Frémat,

D. Ballereau

Co-rotating Circumstellar Clouds around Be Stars . . . . . . . . . . .

L. A. Balona

Apparent Variation of $v \sin i$ and Rotational Modulation in Be Stars . .

C. Neiner, S. Jankov, M. Floquet, A.M. Hubert

How Frequent is Evolutionary Spin-Up in Binary Be Stars? . . . . . . . . .

M. Maintz, Th. Rivinius, S. Štefl, O. Stahl

Rotation of B[e] Supergiants, Luminous Blue Variables and Wolf-Rayet

Stars (Invited Review) . . . . . . . . . . . . . .

$P$. Eenens

Ionization Structure in Equatorial Winds of B[e] Supergiants . . . . .

M. Kraus, H.J.G.L.M. Lamers

The Analysis of the Rotating Disk of some Southern Galactic B[e] Stars

M. Borges Fernandes, H.J.G.L.M. Lamers, M. Kraus, F.X. de Araújo

The Erupting Star in HD5980: A Rapid Rotator ?

L. Georgiev, G. Koenigsberger

\subsection{Rotation in Pre-Main Sequence Stars}

The Rotation of Low-Mass Pre-Main-Sequence Stars (Invited Review)

R.D. Mathieu

On the Relationship Between Stellar Rotation and Radius in Young

Clusters . . . . . . . . . . . . . . . .

L.M. Rebull, S.C. Wolff, S.E. Strom, R.B. Makidon

A Rotational Period Study of a Large Sample of Pre-Main Sequence stars in NGC 2264 . . . . . . . . . . . . . . . . . . . .

M. Lamm, C.A.L. Bailer-Jones, R. Mundt, W. Herbst

\subsection{Rotation in Low Mass Stars, in Red Giants and HB Stars}

Rotational Evolution of Intermediate and Low Mass Main Sequence Stars

(Invited Review) . . . . . . . . . . . . . .

J.R. Stauffer

Rotation of M and L Dwarfs from High Resolution Infrared Spectra .

K.H. Hinkle, L. Wallace, J. Valenti, T. Tsuji

Measurements of Differential Rotation in Cool Stars . . . . . . . . .

A. Reiners, J.H.M.M. Schmitt

The Rotation of Red Giants and Horizontal Branch Stars (Invited

Review) . . . . . . . . . . . . . . .

J.R. De Medeiros 
Post Main-Sequence Changes in Rotational Velocities H.A. Abt

\subsection{Rotation in Binaries}

Observations of Rotation in Binary Stars (Invited Review) . . . . . . 156 G. Koenigsberger

Is there a Connection Between Non-Synchronous Rotation and X-Ray Emission in Massive Binary Systems ? . . . . . . . . . . 163

S. Haro, J.A. Juárez, G. Koenigsberger

A 10-Day Binary System Orbiting the Be Star 66 Oph . . . . . . . . . 166

S. Stefl, P. Hadrava, D. Baade, Th. Rivinius, M. Maintz, O. Stahl

Rotational Velocities of S-Type Symbiotic Stars . . . . . . . . . . . . . . 168

F.C. Fekel, K.H. Hinkle, R.R. Joyce

WIYN Open Cluster Study: Tidal Interactions in Solar type Binaries . 170

S. Meibom, R.D. Mathieu

\subsection{Interferometry, Pulsation and Asteroseismology}

Direct Observations of Rotationally Distorted Stars (Invited Review)

G.T. van Belle, D.R. Ciardi, R.R. Thompson, R.L. Akeson

Modelling Stellar Rotation for Optical Long Baseline Interferometry . . 187

A. Domiciano de Souza, J. Zorec, S. Jankov, F. Vakili, L. Abe

Probing Rotation in Pulsating Stars from Space (Invited Review) . 189

J.M. Matthews

Derivation of the Rotational Frequency of Massive Stars from Seismic

Studies ......................

C. Aerts, R. Scuflaire, A. Thoul

DISCUSSION: Observations of Rotating Stars . . . . . . . . . . 205

D.J. Lennon

\section{SESSION 2. ROTATION IN RELATION WITH} ABUNDANCES AND MAGNETIC FIELDS

\subsection{Chemical Abundances and Rotation}

Abundance Anomalies and Rotation in Main Sequence OB Stars (Invited Review) ................... 209

A. Herrero, D.J. Lennon

CNO Abundances in Magellanic Cloud OB Supergiants . . . . . . . . . 218 P.A. Crowther, C.J. Evans 
Effects of Rotation in Low-Metallicity Stars . . . . . . . . . . . . . . . . 220

S.R. Heap, T. Lanz

CNO abundances in Early Type Be Stars . . . . . . . . . . . . . . .

Y. Frémat, J. Zorec, R. Levenhagen, N. Leister, A.-M. Hubert,

M. Floquet, C. Neiner

Chemical Composition of the Pole-On Be Star HD 120991 . . . . . . . .

Y. Frémat, J. Zorec, A.-M. Hubert

The Impact of Stellar Rotation on the Nitrogen Abundance Gradient from

OB Stars in the Galactic Disk . . . . . . . . . . . . . . .

S. Daflon, K. Cunha

Rotation and Abundances in Red Giants (Invited Review) . . . . . . 228

B. Barbuy

Lithium and Rotation in Pop I stars . . . . . . . . . . . . . . . . 234

S.V. Mallik

Rotation and Lithium Abundances in Evolved Stars . . . . . . . . . .

A. Lèbre, P. de Laverny, J.D. do Nascimento Jr., J.R. de Medeiros

Li Enrichment, Mass Loss, and CN Abundances in High Rotating K Giants

N.A. Drake, R. de la Reza, L. da Silva, D.L. Lambert

HD 3980: a New "Li-spotted" roAp Star . . . . . . . . . . . . . . . .

N.S. Polosukhina, N.A. Drake, M. Hack, R. de la Reza, D. Shakhovskoy

Lithium Abundances in Fast Rotating Bright Giant Stars . . . . . . . . 246

J.D.Jr do Nascimento, A. Lèbre, R. Konstantinova-Antova,

J.R. de Medeiros

\subsection{Magnetic Activity and Rotation}

Rotation and Magnetic Activity in Brown Dwarfs (Invited Review) .

G. Basri

Rotation and Magnetic Fields of Solar-Like Stars (Invited Review) .

J.-F. Donati

A Chandra Look at the Old Open Cluster M67 . . . . . . . . . .

M. van den Berg, G. Tagliafferi, T. Belloni, F. Verbunt

Rotation and Properties of A- and B-Type Chemically Peculiar Stars

(Invited Review) . . . . . . . . . . . . . .

G. Mathys

Fossil Magnetospheres Confront Newborn Dynamos in the Rapid Braking

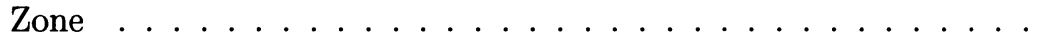

T.R. Ayres

Rotation, Convection, Activity and Lithium in Single G and K giants .

R.K. Konstantinova-Antova 
Magnetic cycles and rotation in active late-type stars . . . . . . .

I. Tuominen, S.V. Berdyugina, M.J. Korpi

X-Ray Activity and Rotation on Bright Late-Type Giants and Supergiants

292

S. Covino, G. Tagliaferri, J.R. de Medeiros

Surface Differential Rotation of Evolved Fast Rotators . . . . . . . . . .

P. Petit, J.-F. Donati, The MuSiCoS collaboration (G.A. Wade,

J.D. Landstreet, T.A.A. Sigut, S.L.S. Shorlin, S. Bagnulo, T. Lüftinger,

S. Strasser, J.M. Oliveira, M. Aurière, F. Lignières, D. Mouillet,

F. Paletou)

DISCUSSION: Rotation in Relation with Abundances and Magnetic Fields

S. Vauclair

\section{SESSION 3. ROTATION, SOLAR AND STELLAR PHYSICS}

\subsection{The Solar Interior}

The internal solar rotation from helioseismology (Invited Review) . .

J. Christensen-Dalsgaard

Dynamical Processes Induced by the Internal Solar Rotation . . . . . .

S. Turck-Chièze

The Eddington Mission . . . . . . . . . . . . . . .

I. Roxburgh, F. Favata

Solar Differential Rotation Revealed by Helioseismology and Simulations of Deep Shells of Turbulent Convection . . . . . . . . . . . . .

J. Toomre, A.S. Brun

Gravitational Distortions of the Shape of the Sun: Constraints on the Models .......................

J.P. Rozelot, S. Lefebvre

Coronal Activity on Rapidly-Rotating Solar-Like Stars: Chandra Observations of ER Vul . . . . . . . . . . . . . . . .

A. Brown, J.M. Brown, R.A. Osten, T.R. Ayres, E. Guinan

\subsection{Physical Processes in Rotating Stars}

Rotational Transport Processes (Invited Review) . . . . . . . . . . 336 S. Talon

The Small-Péclet-Number Approximation for Radiative Zones and its Application to Shear Layer Instabilities . . . . . . . . . . . .

F. Lignières

On Rotationally Driven Meridional Flows in Stars . . . . . . . . . .

P. Garaud 
The Diffusion-Circulation Coupling in Stellar Radiative Zones:

2D Simulation . . . . . . . . . . . . . . . .

S. Théado, S. Vauclair

Angular Momentum Transport and Mixing by Magnetic Fields (Invited

Review) ....................

H.C. Spruit

Three Single Stars (See How They Spin) (Invited Review) . . . . .

P. Charbonneau

Simulations of Core Convection Dynamos in Rotating A-type Stars . . .

M. Browning, A.S. Brun, J. Toomre

Multidimensional Hydrodynamic and Hydrostatic Stellar Models (Invited

Review) . . . . . . . . . . . . . . .

R.G. Deupree

Looking Deep Within an A-type Star: Core Convection Under the Influence of Rotation . . . . . . . . . . . . . . .

A.S. Brun, M. Browning, J. Toomre

Evolution of Rotation in Binaries: Physical Processes (Invited Review)

M. Rieutord

Interactions between Rotation and Pulsation (Invited Review) . . . . 404

R. Townsend

Oscillations of Fast Rotating Stars: p-Modes in Centrifugally Flattened

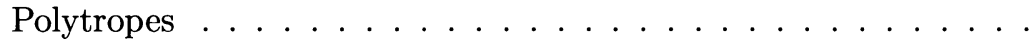

F. Lignières, M. Rieutord

\section{SESSION 4. ROTATION AND STELLAR EVOLUTION}

\subsection{Low and Intermediate Mass Stars}

Pre-Main Sequence Evolution of Rotating Stars in Relation with Observations of Young Clusters (Invited Review) . . . . . . . .

S. Sofia, S.A. Barnes

Activity, Rotation And Convection in Orion: Are the Data Inconsistent with the MS Activity-Rossby Number Relation? . . . . . . . . . .

E. Flaccomio, G. Micela, N. Pizzolato, S. Sciortino, P. Ventura

The Evolution of Angular Momentum of Intermediate Mass Stars: From the Birthline to the Main Sequence . . . . . . . . . . . . .

S.C. Wolff, S.E. Strom, L.A. Hillenbrand

Rotating Models of Low Mass Giants: Rotational Evolution and Surface Abundance Anomalies . . . . . . . . . . . . . . . . .

J. Chanamé, M. Pinsonneault, D. Terndrup

Rotating Models for Evolved Low-Mass Stars (Invited Review) . . . .

C. Charbonnel, A. Palacios 
Problems with the Rotational Models: Observational Constraints . . . .

S.C. Balachandran

Angular momentum evolution in $\mathrm{M} 67 \ldots \ldots \ldots \ldots$

C. Melo, L. Pasquini, J.R. De Medeiros

Constraints on mixing processes from abundance anomalies . . . . . .

G. Michaud, J. Richer, O. Richard (Invited Review)

Mixing and the $s$-process in rotating AGB stars (Invited Review) . .

F. Herwig, N. Langer, M. Lugaro

Morphology of Planetary Nebulae-Possible Effects of Rotation on Stellar Ejecta (Invited Review) . . . . . . . . . . . . .

S. Kwok

\subsection{Massive Stars}

Mass Loss and Stellar Rotation (Invited Review) $\ldots \ldots \ldots \ldots \ldots$

H.J.G.L.M. Lamers

What Ejected Nebulae around Evolved Massive Stars Tell us of the Rotation of their Central Star (Invited Review) . . . . . . .

A. Nota

Evolution of Massive Stars with Rotation and Mass Loss (Invited Review) . . . . . . . . . . . . . . . .

A. Maeder, G. Meynet

Dynamical Shear Instability

R. Hirschi, A. Maeder, G. Meynet

A Comparison of O Spectral Types with Nonrotating and Rotating

Evolutionary Models . . . . . . . . . . . . .

N.R. Walborn, D.J. Lennon

Rotation and Mass Ejection: the Launching of Be-Star Disks (Invited Review) . . . . . . . . . . . . . . .

S.P. Owocki

The Effects of Field-Aligned Rotation on the Magnetically Channeled Line-Driven Winds . . . . . . . . . . . . . . . . . .

A. ud-Doula, S. Owocki

Inhibition of Wind-Compressed Disk Inhibition in Optically Thick Winds

K.G. Gayley

Formation and Dissipation of Viscous Disks around Be Stars . . . . .

A.T. Okazaki

Binary evolution models with rotation (Invited Review) . . . . . . .

N. Langer, S.-C. Yoon, J. Petrovic, A. Heger 
DISCUSSION: Rotation and Stellar Evolution

I.W. Roxburgh, Chairman

\section{SESSION 5. FINAL STAGES, NUCLEOSYNTHESIS}

Rotation and Cataclysmic Variables (Invited Review) . . . . . . . . .

S. Starrfield, E.M. Sion, P. Szkody

White Dwarf Rotation: Observations and Theory (Invited Review) 561

S.D. Kawaler

Effects of Rotation on Helium Accreting White Dwarf . . . . . . . .

S.-C. Yoon, N. Langer

The Rotational Velocity of Helium-rich Pre-White Dwarfs . . . . . . . .

T. Rauch, S. Köper, S. Dreizler, K. Werner, U. Heber, I. Neill Reid

The Enigma of AA Dor ... . . . . . . . . . . . . . . . .

T. Rauch, K. Werner

Hubble Space Telescope Ultraviolet Spectroscopy of Central Stars of the LMC Planetary Nebulae . . . . . . . . . . . . . . .

A. Arrieta, L. Stanghellini, R.A. Shaw, D. Karakla

The effects of Rotation on Wolf-Rayet Stars and on the Production of

Primary Nitrogen by Intermediate Mass Stars . . . . . . . . . .

G. Meynet, M. Pettini (Invited Review)

The Effects of Stellar Yields with Rotation on Chemical Evolution Models

L. Carigi, M. Pettini

Presupernova Evolution of Rotating Massive Stars and the Rotation Rate of Pulsars (Invited Review) . . . . . . . . . . . .

A. Heger, S.E. Woosley, N. Langer, H.C. Spruit

Supernovae, Gamma-Ray Bursts and Stellar Rotation (Invited Review)

S.E. Woosley, A. Heger

DISCUSSION: Final Stages, Nucleosynthesis

N. Langer, Chairman

Summary and Concluding Remarks (Invited Review) . . . . . . . 616 J.-P. Zahn

Author Index 